\title{
Asymmetry of motifs conservation within composite elements
}

\author{
Victor Levitsky \\ ICG SB RAS, Novosibirsk, Russia \\ levitsky@bionet.nsc.ru \\ Tatyana Merkulova \\ ICG SB RAS, Novosibirsk, Russia \\ merkulova@bionet.nsc.ru
}

\author{
Elena Zemlyanskaya \\ ICG SB RAS, Novosibirsk, Russia \\ ezemlyanskaya@bionet.nsc.ru
}

\author{
Dmitry Oshchepkov \\ ICG SB RAS, Novosibirsk, Russia \\ diman@bionet.nsc.ru
}

\begin{abstract}
The cooperative binding to genomic DNA of at least two transcription factors is the widespread mechanism of transcription regulation. Cooperating TFs are recognized through the analysis of co-occurrence of their binding sites. Recently we proposed Motifs Co-Occurrence Tool (MCOT) package that allowed predicting for a single ChIP-seq dataset composite elements (CEs), i.e. overrepresented pairs of spaced or overlapped motifs. Now we updated MCOT package and specified prediction of CEs with one of participating motifs possessing higher conservation than another one. Additionally, for each predicted $\mathrm{CE}$ we tested the hypothesis on the significance of asymmetry toward one or other motif. Hence, MCOT package now provides deeper insight in mechanism of cooperative action of TFs in gene regulation.
\end{abstract}

Keywords - chromatin immunoprecipitation, transcription factor binding site recognition, conservation of motifs, motifs cooccurrence

Motivation and aim

\section{Motivation}

Tissue-, cell- and stage-specific regulation of gene expression is produced through multiple interactions of transcription factors (TFs) with respective regulatory elements called binding sites (BSs) or motifs. Typically, each TF functions in tight cooperation with other TFs [1]. One of the major mechanisms of TF-TF pair binding to DNA consists in preliminary DNA binding of one TF and subsequent binding to TF-DNA complex of other TF. Thus, we may expect that behaviour of two TF is asymmetric. Chromatin immunoprecipitation followed by sequencing (ChIP-seq) analysis becomes the gold standard for protein/DNA-binding annotation at the whole genome level. In particular, ChIP-seq approach has been widely applied for annotation of TFs binding sites (motifs). The standard analysis pipeline at the final stage proposed application of de novo motif search tool, e.g. [2]. We recently proposed the Motifs Co-Occurrence Tool (MCOT) package that allowed predicting spaced as well as overlapped pairs of co-occurred motifs (composite elements, CEs) for a single ChIP-seq dataset [3]. A specific feature of MCOT is the analysis of the range of conservation for each motif in a $\mathrm{CE}$.

Aim

In the current study, we demonstrated an example of the updated MCOT package application. The analysis of motifs conservation within pairs of co-occurred motifs allowed recognizing a specific type of CEs with asymmetric conservation toward either an Anchor or Partner motif.

\section{Methods}

We applied MCOT package as described earlier [3]. This tool annotates pairs of overrepresented motifs, i.e. composite elements (CE). Input data of MCOT comprise the peak dataset in FASTA format, the nucleotide frequency matrix for Anchor motif (that respected to potential BSs of immunoprecipitated TF), and either a Partner motif or the list of potential Partner motifs extracted from Hocomoco human or mouse collections [4].

MCOT classifies CEs according to mutual orientation of motifs, e.g. for heterotypic CEs there are four distinct orientations (Fig. 1A). There are three distinct cases of mutual locations: Full/Partial overlaps and Spacer, consequently MCOT uses five computation flows (Full, Partial, Overlap, Spacer and Any, Fig. 1B). MCOT applies the recognition model of Position Weight Matrix (PWM) for mapping. For each hit of PWM the false positive rates (FPR) for a wholegenome dataset of promoters, so that $-\log _{10}[\mathrm{FPR}]$ value reflects the conservation of motif. For each computation flow, MCOT calculates p-values of Fisher's exact tests that estimates the CE significances and the significance of asymmetry (Fig. 1, C-G).

We modified the original MCOT algorithm [3] to estimate the asymmetry within predicted CEs as follow. We classified all them into two classes with more conservative Anchor or Partner motifs (see Fig. 1, C).

$\left\{-\log _{10}\left[\mathrm{FPR}_{\text {ANCHOR }}\right]>-\log _{10}\left[\mathrm{FPR}_{\text {PARTNER }}\right]\right\}$ (1a)

$\left\{-\log _{10}\left[\mathrm{FPR}_{\text {ANCHOR }}\right] \leq-\log _{10}\left[\mathrm{FPR}_{\text {PARTNER }}\right]\right\}(1 \mathrm{~b})$

We classified the conservation of each motif within the ranges of 12 conservation levels, [<3.5], [3.5..3.7], [3.7..3.9] etc. up to [5.3..5.5] and [>5.5]. We computed the counts of CEs from the foreground and background datasets $\mathrm{Obs}_{\mathrm{i}, \mathrm{j}}$ and $\operatorname{Exp}_{\mathrm{i}, \mathrm{j}}$ that had distinct combinations of conservation levels. Here indices $\mathrm{i}$ and $\mathrm{j}$ denote Anchor and Partner motifs, resectively.Finally, the per mille measure transforms the absolute $\mathrm{CE}$ counts to relative ones as follow: $\left.\left\{1000 * \mathrm{Obs}_{\mathrm{i}, \mathrm{j}} / \mathrm{Obs}\right)\right\}$ and $\left\{1000 * \mathrm{Exp}_{\mathrm{i}, \mathrm{j}} / \operatorname{Exp}\right\}$.

\section{Results}

We illustrate calculation of CE asymmetry with the ChIP-seq data for FoxA2 in mouse liver [5]. In this study, besides the enrichment of the Anchor FoxA2 motif authors revealed its co-occurrence with Partner HNF1 motif. MCOT analysis confirmed this co-occurrence, but also found the significant asymmetry in predicted CEs toward Partner HNF1 $\beta$ motif ( $p$ $<2 \mathrm{E}-28$ and $\mathrm{p}<4 \mathrm{E}-17$ for CEs with an overlap of motifs and with a spacer). Fig. $1 \mathrm{H}$ shows the difference between relative frequencies of observed and expected CEs with overlap of FoxA2 and HNF1 $\beta$ motifs (see Methods).

The recent version of MCOT package is available at https://gitlab.sysbio.cytogen.ru/academiq/moot-kernel.

\section{Acknowledgment}

The work was supported by Russian Foundation for Basic Research (project \# 18-29-13040) and State Budget Project 0324-2019-0040. 


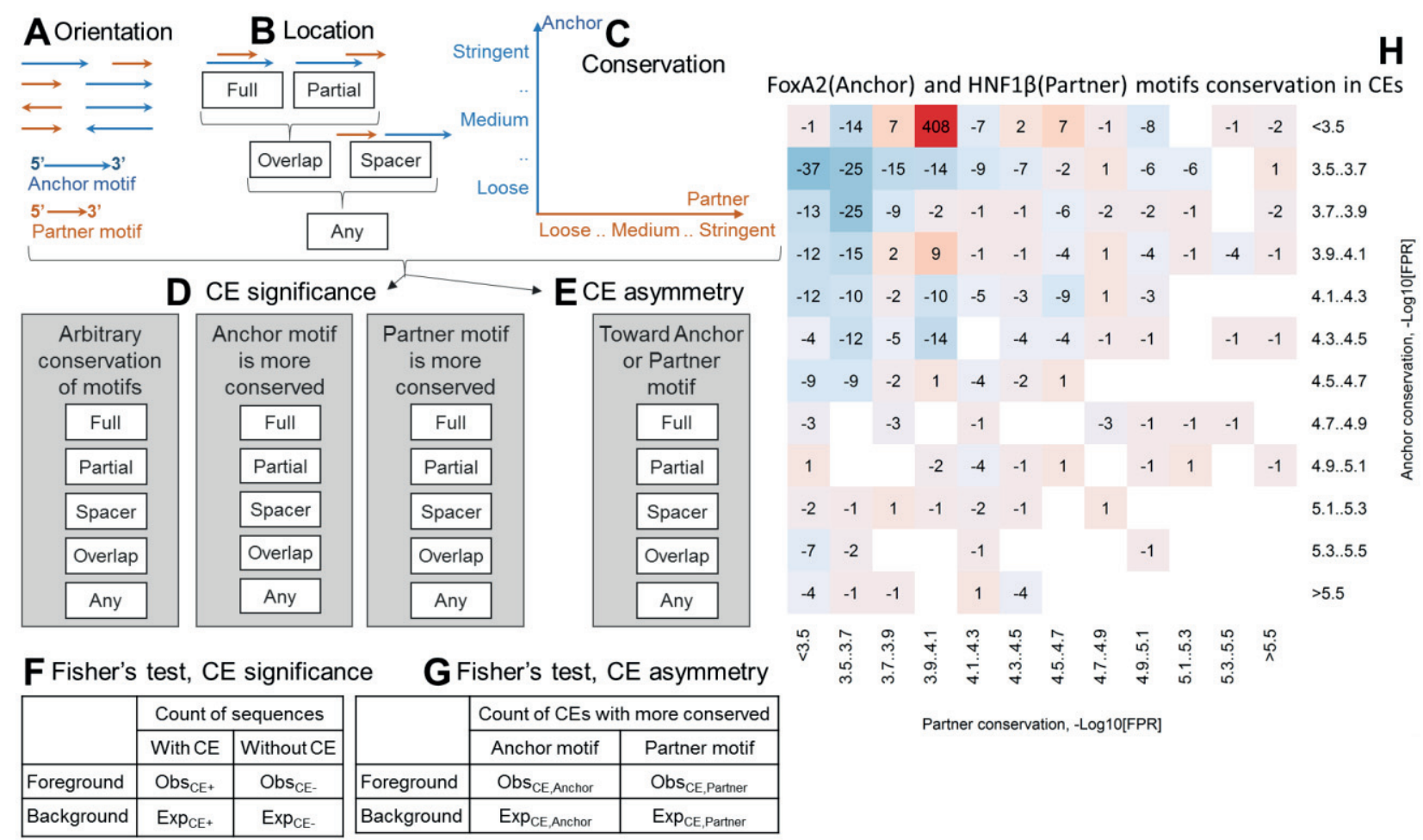

Fig. 1. MCOT package basic concept (A-G) and example analysis of asymmetry within predicted CEs FoxA2/HNF1ß. (H). A, B and C. MCOT classifies all CEs according to mutual orientation, location and conservation of two motifs; the next analysis ( $\mathbf{D}$ and $\mathbf{E})$ is subdivided on five computation flows (Full, Partial, Overlap, Spacer and Any), that were defined according mutual location of motifs (B). For each potential CE we compute (1) it significance with no respect to conservation of motifs or $(2,3)$ two significances for co-occurred pairs with one of motifs (Anchor or Partner) with more conserved hits than for other one (D); The asymmetry between Anchor and Partner motifs can be significant toward one of these motifs. MCOT constructs $2 \times 2$ contingency tables for calculation of significance of CEs (F) and CE asymmetry (G). To compute CE significance $(\mathbf{F})$, we take peaks with hits of both motifs and compare fractions of peaks/permuted sequences that contained CEs. To compute the CE asymmetry $(\mathbf{G})$ we compile two lists of all CEs for peaks and permuted sequences, and compare the fractions of CEs with more conserved Anchor and Partner motifs. H. The color shows the difference between observed (peaks) and expected (permuted sequences) relative abundances of CEs with an overlap of Anchor FoxA2 (axis Y) and Partner HNF1 $\beta$ (axis X) motifs for ChIP-seq data from liver [5], in per mille. FoxA2 and HNF1 $\beta$ motifs we derived from Homer de novo motif search [2] and Hocomoco mouse core collection [4]

\section{REFERENCES}

[1] Reiter F. et al. (2017) Combinatorial function of transcription factors and cofactors, Curr. Opin. Genet. Dev., 43:73-81.

[2] Heinz S., et al. (2010) Simple combinations of lineagedetermining transcription factors prime cis-regulatory elements required for macrophage and $\mathrm{B}$ cell identities. Mol Cell., 38(4), 576-589.
[3] Levitsky V. et al. (2019) A single ChIP-seq dataset is sufficient for comprehensive analysis of motifs cooccurrence with MCOT package, Nucleic Acids Res., 47(21): e139.

[4] Kulakovskiy I.V. et al. (2018) HOCOMOCO: expansion and enhancement of the collection of transcription factor binding sites models. Nucleic Acids Res., 46(D1), D252D259.

[5] Wederell E.D., et al. (2008) Global analysis of in vivo Foxa2-binding sites in mouse adult liver using massively parallel sequencing, Nucleic Acids Res., 36(14):45494564. 